\title{
Two-Reduction of the Super-KP Hierarchy
}

\author{
I. N. McArthur \\ Department of Physics, The University of Western Australia, Nedlands, 6009, Australia
}

Received October 14, 1992

\begin{abstract}
Recursion relations are established for the residues of fractional powers of a two-reduced super-KP operator making use of the Baker-Akhiezer function. These show the integrability of the two-reduced even (or bosonic) flows of the super-KP hierarchy. Similar recursion relations are also proven for the residues of operators associated with the odd (or fermionic) flows of the Mulase-Rabin super-KP hierarchy. Due to the presence of a spectral parameter and its fermionic partner in the BakerAkhiezer function, these recursion relations should be relevant to any attempt to prove or disprove a recent proposal that the integrable hierarchy underlying two-dimensional quantum supergravity is the Mulase-Rabin super-KP hierarchy.
\end{abstract}

\section{Introduction}

A surprising link has been established between the quantum theory of two-dimensional gravity and integrable hierarchies of nonlinear equations of the KdV type [1]. It is therefore of considerable interest to discover whether an integrable system underlies two-dimensional quantum supergravity and its topological counterpart. Recent study of a plausible superloop equation [7] has suggested that the Mulase-Rabin super-KP (SKP) hierarchy $[11,6]$ (or a reduction thereof) is a contender for this role. Further study of this system is therefore warranted.

In this paper, a two-reduced SKP operator is studied. Making use of the BakerAkhiezer function of the Mulase-Rabin SKP hierarchy, identities and recursion relations satisfied by fractional powers of the reduced operator are established, which show that the two-reduced even (or bosonic) flows of the SKP hierarchy are consistent and Hamiltonian. This Hamiltonian structure has been found previously by Oevel and Popowicz [3] using the Lax formulation of the two-reduced even flows. However, the approach taken in this paper is perhaps more relevant to any attempt to prove or disprove the speculation that the integrable system underlying two-dimensional quantum supergravity is a reduction of the Mulase-Rabin SKP hierarchy [7, 8]. This is because of the appearance of a spectral parameter and its fermionic partner via the Baker function, as discussed further in the conclusion. 
In Sect. 2, the Mulase-Rabin SKP hierarchy is briefly reviewed, along with a super-residue formula which is central to the results of this paper. This is applied in Sect. 3 to the proof of recursion relations for residues of fractional powers of a two-reduced SKP operator, and these are used to show the Hamiltonian integrability of the two-reduced even (or bosonic) flows of the SKP hierarchy. Using the Baker function approach, it is also possible to establish identities and recursion relations for the residues of operators which appear in relation to the odd (or fermionic) flows of the Mulase-Rabin SKP hierarchy. This is done in Sect. 4. The conclusion contains an appraisal of the significance of these results to any attempt to establish a link between two-dimensional supergravity and the Mulase-Rabin SKP hierarchy. Calculational details are contained in the appendices.

\section{The Mulase-Rabin SKP Hierarchy}

The SKP hierarchies of nonlinear equations are formulated in terms of the super pseudo-differential operator

$$
Q=D+\hat{q}_{0}(X)+q_{1}(X) D^{-1}+\hat{q}_{2}(X) D^{-2}+\ldots
$$

defined on a (1/1) superspace with coordinates $X=(x, \theta)$. Here, $D=\partial_{\theta}+\theta \partial_{x}$ and $D^{-n}(n>0)$ is defined by $D^{-2 n}=\partial^{-n}$ and $D^{-(2 n+1)}=D \partial^{-(n+1)}$, where $\partial^{-n}=$ $\partial_{x}^{-n}$ is the usual pseudo-differential operator. Hatted superfields are Grassmann-odd. If the superfields $\hat{q}_{0}(X)$ and $q_{1}(X)$ sastisfy the constraint $D \hat{q}_{0}+2 q_{1}=0$, then [2] there exists a pseudo-differential operator

$$
S=\mathbb{1}+\hat{s}_{1}(X) D^{-1}+s_{2}(X) D^{-2}+\ldots
$$

such that

$$
\hat{Q}=S D S^{-1} \text {. }
$$

The even (or bosonic) flows of the SKP hierarchy parameterized by the Grassmanneven parameters $t_{n}, n \geq 1$, can be given in the Lax form

$$
\frac{\partial \hat{Q}}{\partial t_{n}}=\left[\left(\hat{Q}^{2 n}\right)_{+}, \hat{Q}\right]=\left[\hat{Q},\left(\hat{Q}^{2 n}\right)_{-}\right],
$$

where $\left(\hat{Q}^{2 n}\right)_{+}$denotes the differential-operator part of $\hat{Q}^{2 n}$ and $\left(\hat{Q}^{2 n}\right)_{-}=\hat{Q}-\left(\hat{Q}^{2 n}\right)_{+}$. In terms of $S$ the flows are expressed

$$
\frac{\partial S}{\partial t_{n}}=-\left(S \partial^{n} S^{-1}\right)_{-} S
$$

and are integrable [2] in the sense that $\left[\frac{\partial}{\partial t_{n}}, \frac{\partial}{\partial t_{m}}\right] S=0$.

The SKP hierarchy admits several different types of integrable odd (or fermionic) flows (see [6] for a review of these and an explanation of their geometric significance). Here, the Mulase-Rabin hierarchy $[11,6]$ is considered, although the techniques of this paper can be applied to the Manin-Radul hierarchy [2] with minor modification. The Mulase-Rabin flows with respect to Grassmann-odd parameters $\tau_{n-\frac{1}{2}}, n \geq 1$, are
given by

$$
\frac{\partial S}{\partial \tau_{n-\frac{1}{2}}}=-\left(S \partial^{n-1} \partial_{\theta} S^{-1}\right)_{-} S
$$


Unlike the Manin-Radul SKP hierarchy, the odd flows do not admit a Lax formulation purely in terms of the operator $\hat{Q}$, rather

$$
\frac{\partial \hat{Q}}{\partial \tau_{n-\frac{1}{2}}}=-\left\{\hat{Q},\left(S \partial^{n-1} \partial_{\theta} S^{-1}\right)_{-}\right\}
$$

The Baker-Akhiezer function of the Mulase-Rabin SKP hierarchy is

$$
w(\lambda, \zeta ; X, t, \tau)=S \exp \left(\sum_{n \geq 1}\left(t_{n} \lambda^{n}+\tau_{n-\frac{1}{2}} \zeta \lambda^{n-1}\right)+x \lambda+\theta \zeta\right),
$$

where the bosonic spectral parameter $\lambda$ has a fermionic partner $\zeta$. For the purposes of this paper, it will suffice to consider the truncated version

$$
\tilde{w}(\lambda, \zeta ; X)=S e^{x \lambda+\theta \zeta} .
$$

A related function is

$$
\tilde{w}^{*}(\lambda, \zeta ; X)=\left(S^{-1}\right)^{*} e^{-x \lambda-\theta \zeta},
$$

where $\int d X \phi(X) S \psi(X)=\int d X\left(S^{*} \phi(X)\right) \psi(X)$ for superfields $\phi$ and $\psi$. Also important will be the following identity $[14,8]$ : if $P$ and $T$ are super pseudodifferential operators with grading $p$ and $t$ respectively, then

$$
\frac{1}{2 \pi i} \oint d \lambda \int d \zeta\left(P e^{x \lambda+\theta \zeta}\right)\left(T^{\prime *} e^{\left.-x^{\prime} \lambda-\theta^{\prime} \zeta\right)}=(-1)^{(p+t+1)}(P T)_{-} \hat{\delta}\left(X, X^{\prime}\right)\right.
$$

where primed operators act on the variable $X^{\prime}$, and $\hat{\delta}\left(X, X^{\prime}\right)=\delta\left(x, x^{\prime}\right)\left(\theta-\theta^{\prime}\right)$ is the supersymmetric delta function. This is easily established by direct calculation. If $\operatorname{Res}(P T)$ denotes as usual the coefficient of $D^{-1}$ in the super pseudo-differential operator $P T$, then the following super-residue formula applies:

$$
\frac{1}{2} \operatorname{Res}(P T)=(-1)^{(p+t+1)} \lim _{X \rightarrow X^{\prime}} \frac{1}{2 \pi i} \oint d \lambda \int d \zeta\left(P e^{x \lambda+\theta \zeta)}\right)\left(T^{* *} e^{-x^{\prime} \lambda-\theta^{\prime} \zeta}\right) .
$$

This follows from (2.10) using the result

$$
\lim _{X \rightarrow X^{\prime}}(P T)_{-} \hat{\delta}\left(X, X^{\prime}\right)=\frac{1}{2} \operatorname{Res}(P T),
$$

which in turn follows from the definition of the supersymmetric delta function and the corresponding result for nonsupersymmetric residues.

\section{The Two-Reduced SKP Operator}

The two-reduction of the SKP operator $\hat{Q}$ of (2.1) is achieved by the requirement $\hat{Q}^{4}$ is a differential operator $L$, which, using (2.3), is equivalent to

$$
L=S \partial^{2} S^{-1}
$$

with $\left(S \partial^{2} S^{-1}\right)_{-}=0$. Making use of the expansion (2.2) of $S$, it is simple to check that $L$ cannot contain the operators $\partial D$ and $\partial$, so that it has the general form

$$
L=\partial^{2}-\hat{U}(X) D-V(X) .
$$


The two-reduction means that the superfields $s_{n}(X)$ in (2.2) are no longer arbitrary, but satisfy differential relations determined by $L S=S \partial^{2}$. These cannot be solved to give the $s_{n}(X)$ as local functions of $\hat{U}$ and $V$.

The superfields $R_{n}^{(i)}(X), i \geq 0$ will be defined as

$$
2 R_{n}^{(\imath)}(X)=\operatorname{Res}\left(D^{\imath} L^{n-\frac{1}{2}}\right),
$$

where this notation is adopted because the $\hat{R}_{n}^{(0)}$ are the coefficients in the asymptotic expansion of the coincidence limit of the resolvent kernel,

$$
\lim _{X \rightarrow X^{\prime}} \frac{1}{-L+z} \hat{\delta}\left(X, X^{\prime}\right)=\sum_{n=0}^{\infty} \hat{R}_{n}^{(0)}(X) z^{-n-\frac{1}{2}}
$$

(as can be established by expanding the resolvent of $L$ in terms of the resolvent of $\partial^{2}$ in the manner of the appendix of [5] and taking the coincidence limit). As such, the $\hat{R}_{n}^{(0)}$ are local functions of $\hat{U}$ and $V$ and their derivatives. The parameter $z$ is related to the SKP spectral parameter $\lambda$ by $z=\lambda^{2}$. Using the super-residue formula (2.11) with $P=S$ and $T=S^{-1}$, one obtains from $L^{n-\frac{1}{2}}=S \partial^{2 n-1} S^{-1}$ the formula

$$
R_{n}^{(i)}(X)=\lim _{X \rightarrow X^{\prime}}(-1)^{i+1} \frac{1}{2 \pi i} \oint d \lambda \int d \zeta \lambda^{2 n-1} D^{2} \tilde{w}(\lambda, \zeta ; X) \tilde{w}^{*}\left(\lambda, \zeta ; X^{\prime}\right)
$$

The superfields $R_{n}^{(i)}$ of (3.3) satisfy a number of relations as a result of the form (3.2) of $L$, which allows them to be generated recursively using $\hat{R}_{0}^{(0)}=0$. Defining the combinations

$$
\begin{aligned}
& \hat{A}_{n}=\hat{R}_{n}^{(2)}-\frac{1}{2} D R_{n}^{(1)} \\
& B_{n}=R_{n}^{(3)}+D \hat{R}_{n}^{(2)}-\partial R_{n}^{(1)} \\
& C_{n}=\partial R_{n}^{(1)}-(\partial D-\hat{U}) \hat{R}_{n}^{(0)}
\end{aligned}
$$

then they satisfy the differential relations

$$
\begin{aligned}
D \hat{A}_{n} & =-\frac{1}{2} C_{n}, \\
\partial B_{n} & =(\partial D-\hat{U}) \hat{A}_{n}+\frac{1}{2}(D V) \hat{R}_{n}^{(0)},
\end{aligned}
$$

and the recursion relations

$$
\begin{aligned}
\partial \hat{R}_{n+1}^{(0)}= & \frac{1}{4}\left(\partial^{2} D-3 \hat{U} \partial-(D \hat{U}) D-2(\partial \hat{U})\right) R_{n}^{(1)} \\
& +\frac{1}{2} D(\partial D-\hat{U}) \hat{A}_{n}-\left(\frac{1}{2}(\partial V)+V \partial\right) \hat{R}_{n}^{(0)} \\
C_{n+1}= & -\frac{1}{2}(\partial D-\hat{U}) D B_{n}+\left(2 V D+\frac{1}{2}(D V)\right) \hat{A}_{n} \\
& -\frac{1}{2}\left((\partial V)-\frac{1}{2}(D V) D\right) R_{n}^{(1)} \\
& +\frac{1}{2}((D V) \partial+(\partial V) D+(\partial D V)) \hat{R}_{n}^{(0)}
\end{aligned}
$$

These are proven in Appendix A using the representation (3.4) of the $R_{n}^{(2)}$, and involves operating on $\tilde{w}(\lambda, \zeta ; X) \tilde{w}^{*}\left(\lambda, \zeta ; X^{\prime}\right)$ with sums and differences of operators acting at the points $X$ and $X^{\prime}$ before taking the coincidence limit $X \rightarrow X^{\prime}$. It does not require the imposition of the SKP equations on $S$, but relies only on the form (3.2) of the reduced SKP operator $L$. 
Next, the even (or bosonic) flows for the two-reduced SKP operator are considered. By (2.5), all dependence of $S$ on $t_{2 n}$ vanishes, as $\left(S \partial^{2 n} S^{-1}\right)_{-}$vanishes for the tworeduced SKP operator. The remaining even flows take the Lax form

$$
\frac{\partial L}{\partial t_{2 n-1}}=\left[L,\left(L^{n-\frac{1}{2}}\right)_{-}\right] \text {. }
$$

Substituting the form (3.2) for $L$, one finds from the coefficients of $D$ and $\mathbb{1}$ on the right-hand side that

$$
\begin{aligned}
& \frac{\partial \hat{U}}{\partial t_{2 n-1}}=-2 \partial \operatorname{Res}\left(L^{n-\frac{1}{2}}\right)=-4 \partial \hat{R}_{n}^{(0)}, \\
& \frac{\partial V}{\partial t_{2 n-1}}=-2 \partial \operatorname{Res}\left(D L^{n-\frac{1}{2}}\right)+2(\partial D-\hat{U}) \operatorname{Res} L^{n-\frac{1}{2}}=-4 C_{n} .
\end{aligned}
$$

Consistency of the two-reduction with the even SKP flows requires that these nonlinear equations be integrable. The integrability and Hamiltonian nature of these equations has been established in [3] using the Lax formulation (3.6). Below, these results are demonstrated using the identities (I)-(IV). This provides an outlook on the integrability of the two-reduced SKP hierarchy which relates to the Baker function via (3.4), where the appearance of the spectral parameter $\lambda$ and its fermionic partner $\zeta$ should be important in any attempt to relate this integrable system to the superVirasoro constraints which have been conjectured for the partition function of twodimensional quantum supergravity. This is discussed further in the conclusion. Using $\hat{R}_{n}^{(0)}=\operatorname{Res}\left(L^{n-\frac{1}{2}}\right)$ together with $\frac{\delta L}{\delta \hat{U}(X)}=-D$ and $\frac{\delta L}{\delta V(X)}=-\mathbb{1}$, it
follows that

$$
\begin{aligned}
& \frac{\delta \hat{R}_{n+1}^{(0)}(X)}{\delta \hat{U}(X)}=-\left(n+\frac{1}{2}\right) R_{n}^{(1)}, \\
& \frac{\delta \hat{R}_{n+1}^{(0)}(X)}{\delta V(X)}=-\left(n+\frac{1}{2}\right) \hat{R}_{n}^{(0)},
\end{aligned}
$$

(this can also be proved using the identification of the superfields $\hat{R}_{n}^{(0)}$ with the coefficients in the expansion of the coincidence limit of the resolvent of $L$, after the method of [4]). Here, the variational derivatives are defined by $\delta \int d X F[\hat{U}, V]=$ $\int d X \delta \hat{U}(X) \frac{\delta F}{\delta \hat{U}(X)}+\int d X \delta V(X) \frac{\delta F}{\delta V(X)}$ for a functional $F[\hat{U}, V]$ of the superfields $\hat{U}$ and $V$. Thus the Eqs. (3.7) can be put in the Hamiltonian form

$$
\begin{aligned}
& \frac{\partial \hat{U}(X)}{\partial t_{2 n-1}}=\left[\hat{U}(X), H_{n}\right]_{\mathrm{PB}} \\
& \frac{\partial V(X)}{\partial t_{2 n-1}}=\left[V(X), H_{n}\right]_{\mathrm{PB}}
\end{aligned}
$$

with Hamiltonian

$$
H_{n}=-\frac{4}{\left(n+\frac{1}{2}\right)} \int d X \hat{R}_{n+1}^{(0)}(X)
$$


and the (graded) Poisson brackets

$$
\begin{aligned}
& \left\{\hat{U}(X), \hat{U}\left(X^{\prime}\right)\right\}_{\mathrm{PB}}=0 \\
& {\left[\hat{U}(X), V\left(X^{\prime}\right)\right]_{\mathrm{PB}}=\partial \hat{\delta}\left(X, X^{\prime}\right),} \\
& {\left[V(X), \hat{U}\left(X^{\prime}\right)\right]_{\mathrm{PB}}=\partial \hat{\delta}\left(X, X^{\prime}\right),} \\
& {\left[V(X), V\left(X^{\prime}\right)\right]_{\mathrm{PB}}=-(\partial D-\hat{U}(X)) \hat{\delta}\left(X, X^{\prime}\right) .}
\end{aligned}
$$

The Poisson brackets have the correct symmetry properties and obey the super-Jacobi identity. Integrability of the hierarchy (3.7) is equivalent to showing $\left[H_{n}, H_{m}\right]_{\mathrm{PB}}=0$, which is done in Appendix B using the identities (I)-(IV).

To relate these results to the proof of the integrability of (3.7) via the Lax formulation [3], the identities (I)-(IV) ensure the vanishing of the coefficients of the operators $D^{-1}, D^{-2}, D^{-3}$ and $D^{-4}$ respectively on the right-hand side of (3.6).

By setting $V=0$ in (3.2), $L$ becomes the super-KdV operator. This reduction has been considered in [3]. In terms of the approach taken here, consistency of the condition $V=0$ with the two-reduced even flows requires, by (3.7), that $C_{n}$ vanishes, or that $\partial R_{n}^{(1)}=(\partial D-\hat{U}) \hat{R}_{n}^{(0)}$. This is indeed the case for the super-KdV operator $L=\partial^{2}-\hat{U} D$, as was shown in [4].

\section{Recursion Relations Related to Odd Flows}

The Baker-function formalism also allows the determination of recursion relations for the residues of operators which are related to the odd flows (2.6) of the Mulase-Rabin SKP hierarchy (these cannot be seen using the techniques of [4] in which only the resolvent is employed.) If $S$ is the wave operator of the two-reduced SKP operator, in that Eqs. (3.1) and (3.2) are fulfilled, then by carrying out the steps of Appendix A with the quantity $\hat{R}_{n}\left(X, X^{\prime}\right)$ of Eq. (A.1) replaced by

$$
-\frac{1}{2 \pi i} \oint d \lambda \int d \zeta \lambda^{n} \zeta F\left(X, X^{\prime} ; \lambda, \zeta\right),
$$

it is possible to extablish identities of the form (I)-(IV) with $2 R_{n}^{(\imath)}=\operatorname{Res}\left(D^{\imath} L^{n-\frac{1}{2}}\right)$ replaced everywhere by $\operatorname{Res}\left(D^{i} S \partial^{n} \partial_{\theta} S^{-1}\right)$. Note that again this relies only on Eqs. (3.1) and (3.2) satisfied by $S$ and makes no assumption the $S$ obeys the MulaseRabin SKP equations (2.6). Also, the quantities $\operatorname{Res}\left(D^{\imath} S \partial^{n} \partial_{\theta} S^{-1}\right)$ are not local in the superfields $\hat{U}$ and $V$, although they are local in terms of the superfields $s_{n}(X)$ in the expansion (2.2) of $S$. These recursion relations have been checked to low order by hand using the relations between the superfields $s_{n}(X)$ implied by $L S=S \partial^{2}$.

The significance of these recursion relations to the two-reduced (with respect to the parameters for the even flows only) SKP hierarchy is that they are respectively equivalent to the vanishing of the coefficients of the operators $D^{-1}, D^{-2}, D^{-3}$, and $D^{-4}$ on the right-hand side of the equation

$$
\frac{\partial L}{\partial \tau_{n-\frac{1}{2}}}=\left[L,\left(S \partial^{n-1} \partial_{\theta} S^{-1}\right)_{-}\right] .
$$

This shows the consistency of the odd flows with the two-reduction. 
The superfield $V$ cannot be set to zero consistently in the presence of the odd flows, in contrast to the situation for the even flows. This is because, by (4.1), the vanishing of $\frac{\partial V}{\partial \tau_{n+\frac{1}{2}}}$ requires $\partial \operatorname{Res}\left(D S \partial^{n} \partial_{\theta} S^{-1}\right)=(\partial D-\hat{U}) \operatorname{Res}\left(S \partial^{n} \partial_{\theta} S^{-1}\right)$, which is not true for $L=\partial^{2}-\hat{U} D$, as can be verified by explicit computation for small $n$.

\section{Conclusion}

As mentioned in the introduction, any relation between the reduced Mulase-Rabin SKP hierarchy and two-dimensional quantum supergravity is likely to proceed via the spectral parameter $\lambda$ and its fermionic partner $\zeta$ in the Baker function (2.7). To make this clearer, we recall briefly the situation in ordinary (nonsupersymmetric) twodimensional topological quantum gravity, where the partition function is a tau function for the $\mathrm{KdV}$ hierarchy subject to the additional constraint that it satisfies the string equation [1]. These two conditions are equivalent to a set of Virasoro constraints on the partition function $[12,13]$, which have been interpreted in terms of the geometry of moduli space by Kontsevich [9] and Witten [10].

The Virasoro constraints can be traced to conformal transformations of the spectral parameter $\lambda$ of the two-reduced $\mathrm{KP}$ (or KdV) hierarchy, as follows. The coincidence limit $R(x ; z)$ (with $z=\lambda^{2}$ ) of the resolvent of the two-reduced KP operator $\partial_{x}^{2}-u(x)$ satisfies a set of recursion relations

$$
z \partial_{x} R(x ; z)=\frac{1}{4}\left(\partial_{x}^{3}-4 u(x) \partial_{x}-2 u^{\prime}(x)\right) R(x ; z) .
$$

Acting on both sides of the equation with $-\partial_{z} z^{m+1}$ and using the fact that $\mathscr{L}_{m}=$ $-\partial_{z} z^{m+1}$ is a generator of conformal transformations in the parameter $z$ for rank one tensors, the recursion relations can be written

$$
\partial_{x} \mathscr{S}_{m+1} R(x ; z)=\frac{1}{4}\left(\partial_{x}^{3}-4 u(x) \partial-2 u^{\prime}(x)\right) \mathscr{L}_{m} R(x ; z), \quad m \geq-1 .
$$

Via the relation between the resolvent and the Baker function, conformal transformations of $z$ induce transformations of the parameters $t_{n}$ describing the $\mathrm{KdV}$ flows, and this recursion relation becomes a condition on the tau function $\tau$ of the $\mathrm{KdV}$ hierarchy,

$$
\partial_{x}^{2}\left(\tau^{-1} L_{m+1} \tau\right)=\frac{1}{4}\left(\partial_{x}^{3}-4 u(x) \partial-2 u^{\prime}(x)\right) \partial\left(\tau^{-1} L_{m} \tau\right), \quad m \geq-1,
$$

where the $L_{m}$ are Virasoro generators constructed from bilinears in the $t_{n}$. This recursion relation combined with the string equation $L_{-1} \tau=0$ establishes the Virasoro constraints $L_{m} \tau=0, m \geq 0[12,13]$.

In [7], it has been proposed that the partition function for topological supergravity should satisfy a set of super-Virasoro constraints, and analysis of the corresponding superloop equations suggested that the Mulase-Rabin SKP hierarchy is a candidate for the corresponding integrable system. Based on the nonsupersymmetric case outlined above, it could be expected that superconformal transformations of the spectral parameter $\lambda$ and its fermionic partner $\zeta$ in the Baker function of the SKP hierarchy would be related to any super-Virasoro constraints on the partition function, and that recursion relations obeyed by the reduced hierarchy would be an important element of the structure. In this paper, these recursion relations have been at least partly elucidated in relation to the integrability of the reduced hierarchy. However, due to 
the complicated nature of the recursion relations (I)-(IV) and their counterparts for the odd SKP flows found in Sect. 4, there is no obvious sign of any super-Virasoro structure. Probably related, there is also no obvious reason why $\lambda$ and $\zeta$ should be coordinates on a superspace with torsion, as required for a superconformal structure. This presumably would emerge if a formulation of the SKP hierarchy in terms of free superfields on a super-Riemann surface with coordinates $(\lambda, \zeta)$ could be found, analogous to the Grassmannian formulation of the ordinary KP hierarchy [15].

\section{Appendix A}

Here, the proofs of identities (I)-(IV) are given. The fundamental object of interest is

$$
F\left(X, X^{\prime} ; \lambda, \zeta\right)=-\tilde{w}(\lambda, \zeta ; X) \tilde{w}^{*}\left(\lambda, \zeta ; X^{\prime}\right),
$$

where $\tilde{w}$ and $\tilde{w}^{*}$ are defined in (2.8) and (2.9). We also define

$$
\hat{R}_{n}\left(X, X^{\prime}\right)=\frac{1}{2 \pi i} \oint d \lambda \int d \zeta \lambda^{2 n-1} F\left(X, X^{\prime} ; \lambda, \zeta\right),
$$

which, by (2.10) and (3.1), is equivalent to $\left(L^{n-\frac{1}{2}}\right)_{-} \hat{\delta}\left(X, X^{\prime}\right)$. Then, using (2.11),

$$
\lim _{X \rightarrow X^{\prime}} \hat{R}_{n}\left(X, X^{\prime}\right)=\frac{1}{2} \operatorname{Res}\left(L^{n-\frac{1}{2}}\right)=\hat{R}_{n}^{(0)}(X) .
$$

Similarly, if $\mathbf{O}$ is some operator acting on the argument $X$,

$$
\lim _{X \rightarrow X^{\prime}} \mathbf{O} \hat{R}_{n}\left(X, X^{\prime}\right)=\frac{1}{2} \operatorname{Res}\left(\mathbf{O} L^{n-\frac{1}{2}}\right),
$$

The method of proof of the identities is very similar to that employed in [4] for the super-KdV equation using heat kernels. Therefore, only brief details are given. A fundamental identity is

$$
0=\left(L-L^{\prime *}\right) F\left(X, X^{\prime} ; \lambda, \zeta\right),
$$

which follows from $0=\left(\lambda^{2}-\lambda^{2}\right) F\left(X, X^{\prime} ; \lambda, \zeta\right)$.

Taking the contour integral $\lim _{X \rightarrow X^{\prime}} \frac{1}{2 \pi i} \oint d \lambda \int d \zeta \lambda^{2 n-1}$ of (A.3), and using (A.2) and identities such as $\lim _{X \rightarrow X^{\prime}}\left(\mathbf{O}+\mathbf{O}^{\prime}\right) F_{n}\left(X, X^{\prime}\right)=\mathbf{O} \lim _{X \rightarrow X^{\prime}} F_{n}\left(X, X^{\prime}\right)$ and $\lim _{X \rightarrow X^{\prime}}\left(\partial-\partial^{\prime}\right) F_{n}\left(X, X^{\prime}\right)=2 \lim _{X \rightarrow X^{\prime}} \partial F_{n}\left(X, X^{\prime}\right)-\partial \lim _{X \rightarrow X^{\prime}} F_{n}\left(X, X^{\prime}\right)$, one finds

$$
\partial \hat{R}_{n}^{(2)}=\frac{1}{2} D(\partial D-\hat{U}) \hat{R}_{n}^{(0)} .
$$

Similarly, acting on (A.3) with $\left(D-D^{\prime}\right)$ and carrying out the above steps, together with

$$
\begin{aligned}
& \lim _{X \rightarrow X^{\prime}}\left(D-D^{\prime}\right) F_{n}\left(X, X^{\prime}\right) \\
& =2 \lim _{X \rightarrow X^{\prime}} D F_{n}\left(X, X^{\prime}\right)-D \lim _{X \rightarrow X^{\prime}} F_{n}\left(X, X^{\prime}\right), \\
& \lim _{X \rightarrow X^{\prime}}\left(\partial-\partial^{\prime}\right)\left(D-D^{\prime}\right) F_{n}\left(X, X^{\prime}\right) \\
& =4 \lim _{X \rightarrow X^{\prime}} \partial D F_{n}\left(X, X^{\prime}\right)-2 \partial \lim _{X \rightarrow X^{\prime}} D F_{n}\left(X, X^{\prime}\right) \\
& \quad-2 D \lim _{X \rightarrow X^{\prime}} \partial F_{n}\left(X, X^{\prime}\right)+\partial D \lim _{X \rightarrow X^{\prime}} F_{n}\left(X, X^{\prime}\right),
\end{aligned}
$$


one finds

$$
\partial R_{n}^{(3)}=\frac{1}{2}\left(\partial^{2}+\hat{U} D\right) R_{n}^{(1)}-\hat{U} \hat{R}_{n}^{(2)}+\frac{1}{2}(D V) \hat{R}_{n}^{(0)} .
$$

Identities (I) and (II) follow from (A.4) and (A.5).

The recursion relations (III) and (IV) follow by considering the relation

$$
\lambda^{2} F\left(X, X^{\prime} ; \lambda, \zeta\right)=\frac{1}{2}\left(L+L^{\prime *}\right) F\left(X, X^{\prime} ; \lambda, \zeta\right) .
$$

Acting on both sides with $\left(\partial+\partial^{\prime}\right)$, adding the identity $0=-\frac{1}{4}\left(\partial-\partial^{\prime}\right)(L-$ $\left.L^{\prime *}\right) F\left(X, X^{\prime} ; \lambda, \zeta\right)$ and carrying out the above steps gives

$$
\begin{aligned}
\partial \hat{R}_{n+1}^{(0)}= & \frac{1}{4}\left(\partial^{3}+\hat{U} \partial D-(D \hat{U}) \partial+(\partial \hat{U}) D-(\partial D \hat{U})-\frac{1}{2}(\partial V)-V \partial\right) \hat{R}_{n}^{(0)} \\
& +\frac{1}{2}(\hat{U} D-(D \hat{U})) \hat{R}_{n}^{(2)}-\left(\hat{U} \partial+\frac{1}{2}(\partial \hat{U})\right) R_{n}^{(1)}
\end{aligned}
$$

which yields (III) after use of (A.4) and (A.5). Finally, acting on both sides of (A.6) with $\left(\partial+\partial^{\prime}\right)\left(D-D^{\prime}\right)$ and carrying out the above steps (including adding the identity $\left.0=-\frac{1}{4}\left(D-D^{\prime}\right)\left(\partial-\partial^{\prime}\right)\left(L-L^{\prime *}\right) F\left(X, X^{\prime} ; \lambda, \zeta\right)\right)$ yields

$$
\begin{aligned}
& 2 \partial R_{n+1}^{(1)}-\partial D \hat{R}_{n+1}^{(0)} \\
&=-2 \hat{U} \lim _{X \rightarrow X^{\prime}} \partial^{2} F_{n}\left(X, X^{\prime}\right)+\hat{U} D R_{n}^{(3)} \\
&+\frac{1}{4}(6 \hat{U} \partial+2(\partial \hat{U})+4(D V)) \hat{R}_{n}^{(2)} \\
&+\frac{1}{4}\left(2 \partial^{3}-2 \hat{U} \partial D-4(D \hat{U}) \partial-2(\partial D \hat{U})-8 V \partial-4(\partial V)\right) R_{n}^{(1)} \\
&+\frac{1}{4}\left(-\partial^{3} D+\hat{U} \partial^{2}+2(\partial \hat{U}) \partial+\left(\partial^{2} \hat{U}\right)+4 V \partial D-2(D V) \partial\right. \\
&+2(\partial V) D) \hat{R}_{n}^{(0)} .
\end{aligned}
$$

The term $\hat{U} \lim _{X \rightarrow X^{\prime}} \partial^{2} F_{n}\left(X, X^{\prime}\right)$ can be eliminated using $0=\left(L-\lambda^{2}\right) F\left(X, X^{\prime} ; \lambda, \zeta\right)$, giving

$$
\hat{R}_{n+1}^{(0)}=\lim _{X \rightarrow X^{\prime}} \partial^{2} F_{n}\left(X, X^{\prime}\right)-\hat{U} R_{n}^{(1)}-V \hat{R}_{n}^{(0)} .
$$

Combining this with (A.8), (A.7), (A.5) and (A.4) yields the recursion relation (IV).

\section{Appendix B}

Here, it is shown that the Hamiltonians (3.9) are in involution with respect to the Poisson brackets (3.10). Using (3.8), one finds

$$
\left[H_{n}, H_{m}\right]_{\mathrm{PB}}=16 \int d X\left(\left(\partial \hat{R}_{m}^{(0)}\right) R_{n}^{(1)}+C_{m} \hat{R}_{n}^{(0)}\right) .
$$

Substituting the definition (3.5) of $C_{m}$, this is easily seen to be antisymmetric under interchange of $m$ and $n$, as it should be. Applying the recursion relations (III) and (IV) for $\partial \hat{R}_{m}^{(0)}$ and $C_{m}$, the aim is to show that the resulting expression is antisymmetric under the interchange of $m-1$ and $n$, as the recursion relations can then be applied in reverse to obtain

$$
\begin{aligned}
{\left[H_{n}, H_{m}\right]_{\mathrm{PB}} } & =-16 \int d X\left(\left(\partial \hat{R}_{n+1}^{(0)}\right) R_{m-1}^{(1)}+C_{n+1} R_{m-1}^{(0)}\right) \\
& =\left[H_{n+1}, H_{m-1}\right]_{\mathrm{PB}} .
\end{aligned}
$$

Using $H_{0}=0$ (since $\hat{R}_{0}^{(0)}=0$ ), it then follows that $\left[H_{n}, H_{m}\right]_{\mathrm{PB}}=0$, as required. 
To show the antisymmetry under the interchange of $m-1$ and $n$, substitution for $\partial \hat{R}_{m}^{(0)}$ and $C_{m}$ in (B.1) gives

$$
\frac{1}{4}\left[H_{n}, H_{m}\right]_{\mathrm{PB}}=a_{1}+a_{2}+a_{3}+a_{4}+a_{5}
$$

with

$$
\begin{aligned}
& a_{1}=\frac{1}{4} \int d X R_{n}^{(1)}\left(\partial^{2} D-3 \hat{U} \partial-(D \hat{U}) D-2(\partial \hat{U})\right) R_{m-1}^{(1)} \\
& a_{2}=\frac{1}{2} \int d X R_{n}^{(1)} D(\partial D-\hat{U}) \hat{A}_{m-1}-\frac{1}{2} \int d X \hat{R}_{n}^{(0)}(\partial D-\hat{U}) D B_{m-1}, \\
& a_{3}=-\int d X R_{n}^{(1)}\left(V \partial+\frac{1}{2}(\partial V)\right) \hat{R}_{m-1}^{(0)}-\frac{1}{2} \int d X \hat{R}_{n}^{(0)}\left((\partial V)-\frac{1}{2}(D V) D\right) R_{m-1}^{(1)}, \\
& a_{4}=\int d X \hat{R}_{n}^{(0)}\left(2 V D+\frac{1}{2}(D V)\right) \hat{A}_{m-1}, \\
& a_{5}=\frac{1}{2} \int \hat{R}_{n}^{(0)}((D V) \partial+(\partial V) D+(\partial D V)) \hat{R}_{m-1}^{(0)} .
\end{aligned}
$$

Using (II) and (III) and integrating by parts several times,

$$
\begin{aligned}
a_{2}= & \int d X \hat{A}_{n}(\partial D-\hat{U}) \hat{A}_{m-1}-\frac{1}{2} \int d X(D V) \hat{A}_{n} \hat{R}_{m-1}^{(0)} \\
& -\frac{1}{4} \int d X(D V)\left(D R_{n}^{(1)}\right) \hat{R}_{m-1}^{(0)} .
\end{aligned}
$$

Using (I) and the definition (3.5) of $C_{m-1}$,

$$
\begin{aligned}
a_{4}= & -\frac{1}{2} \int d X(D V) \hat{R}_{n}^{(0)} \hat{A}_{m-1}+\frac{1}{2} \int d X(\partial V) \hat{R}_{n}^{(0)} R_{m-1}^{(1)} \\
& +\int d X V\left(-\frac{1}{2} \hat{R}_{n}^{(0)} \partial R_{m-1}^{(1)}+\frac{1}{2}\left(\partial \hat{R}_{n}^{(0)}\right) R_{m-1}^{(1)}\right. \\
& \left.+\hat{R}_{n}^{(0)} \partial D \hat{R}_{m-1}^{(0)}+\hat{U} \hat{R}_{n}^{(0)} \hat{R}_{m-1}^{(0)}\right) .
\end{aligned}
$$

Making use of these expressions and symmetrizing some of the derivatives in $a_{3}$ and $a_{5}$, one easily establishes the antisymmetry of $a_{1}+\ldots+a_{5}$ under the interchange of $m-1$ and $n$.

Acknowledgements. The author is grateful to Dr. C. M. Yung for discussions on integrable systems. This work was supported by an Australian Research Fellowship.

\section{References}

1. Gross, D., Migdal, A.B.: Phys. Rev. Lett. 64, 127 (1990)

Brézin, E., Kazakov, V.A.: Phys. Lett. B 236, 144 (1990)

Douglas, M.R., Shenker, S.H.: Nucl. Phys. B 335, 635 (1990)

Douglas, M.R.: Nucl. Phys. B 238, 176 (1990)

Dijkgraaf, R., Witten, E.: Nucl. Phys. 342, 486 (1990)

2. Manin, Yu.I., Radul, A.O.: Commun. Math. Phys. 98, 65 (1985)

3. Oevel, W., Popowicz, Z.: Commun. Math. Phys. 139, 41 (1990)

4. McArthur, I.N.: Commun. Math. Phys. 148, 177 (1992) 
5. Gelfand, I.M., Dikii, L.A.: Russ. Math. Surveys 30 (5), 77 (1975)

6. Rabin, J.M.: Commun. Math. Phys. 137, 533 (1991)

7. Alvarez-Gaumé, L., Itoyama, H., Manes,. J.L., Zadra, A.: Int. J. Mod. Phys. A 7,5337 (1992)

8. Itoyama, H.: Phys. Lett. B 299, 64 (1993)

9. Kontsevich, M.: Commun. Math. Phys. 147, 1 (1992)

10. Witten, E.: On the Kontsevich model and other models of two-dimensional gravity. IAS preprint HEP-91/24

11. Mulase, M.: J. Diff. Geom. 34, 651 (1991)

12. Dijkgraaf, R., Verlinde, E., Verlinde, H.: Nucl. Phys. B 348, 435 (1991)

13. Fukuma, M., Kuwai, H., Nakayama, R.: Int. J. Mod. Phys. A 6, 1385 (1991)

14. Ueno, K., Yamada, H., Ikeda, K.: Commun. Math. Phys. 124, 57 (1989)

15. Jimbo, M., Miwa, T.: In: Vertex operators in mathematics and physics. Lepowsky, J., Mandelstam, S., Singer, I. (eds.). Berlin, Heidelberg, New York: Springer 1984

Communicated by K. Gawedzki 
